\title{
Sedation for children requiring wound repair: a randomised controlled double blind comparison of oral midazolam and oral ketamine
}

\author{
Paul A Younge, Jason M Kendall
}

\begin{abstract}
Objective-To compare the efficacy of oral ketamine $(10 \mathrm{mg} / \mathrm{kg})$ with oral midazolam $(0.7 \mathrm{mg} / \mathrm{kg})$ in providing sedation for suturing of lacerations.

Method-Prospective, randomised, double blinded trial with consecutive, concealed recruitment of 59 children aged 1 to 7 with wounds requiring local anaesthetic (LA) injection or topical LA with an anxiety score greater than one.

Results-Tolerance to LA injection was better with ketamine $(p=0.029)$ and tolerance to procedure after LA injection showed a trend towards being improved with ketamine $(p=0.067)$. There was no difference in tolerance to LA application or procedure in children receiving topical LA. Time to reach a sedation score of less than four was faster with ketamine (medians 20 versus 43 minutes, $p=0.001$ ) but times from dosing to discharge (medians 105 and 110 minutes) were similar. Inconsolable agitation was reported with midazolam in six cases. Dysphoria was not noted with ketamine. Vomiting was more common with ketamine but not significantly so ( six versus two, $p=0.14$ ). Oxygen desaturations were noted in both groups. Ataxia after discharge was seen in four patients, two in each group. Thirty six per cent of children showed new behavioural disturbances in the two weeks after discharge, more commonly in the midazolam group $(p=0.048)$.

Conclusions-At these doses tolerance to LA injection was better in children receiving ketamine, with fewer behavioural changes noted in the first two weeks. Midazolam at this dose caused dysphoric reactions, which may have affected the results. Continuous pulse oximetry monitoring is required when using these drugs. Vomiting and prolonged ataxia occurred in a few patients.

(Emerg Med f 2001;18:30-33)
\end{abstract}

Keywords: suturing; children; ketamine; midazolam

Children, Bristol

P A Younge

Emergency

Department, Frenchay

Hospital, Bristol

$\mathrm{J}$ M Kendall

Correspondence to: Dr Younge, 30 Burghley Road, Bristol BS6 5BN (sueandpaul@cableinet.co.uk)

Accepted 29 February 2000 replace injected local anaesthetic in over $80 \%$ of wounds in children in our experience. It has limitations concerning wound size, use on extremities and proximity to mucous membranes. However, often these techniques are not enough. Restraint has been used and although there is no evidence showing such experiences have long term effects, behavioural changes suggesting a traumatic experience have been recorded after admission to hospital for surgery ${ }^{2}$ and have been reduced by premedication. ${ }^{4}$

There is no consensus on the best sedative drug for this purpose, its dose or route of administration. The possibilities in this area have been well reviewed. ${ }^{5}$

Perhaps most commonly used is oral midazolam, which has been shown more effective than placebo at a dose of $0.2 \mathrm{mg} / \mathrm{kg}$. ${ }^{6}{ }^{7}$ There is evidence that sedative anxiolytic effects increase up to $0.5 \mathrm{mg} / \mathrm{kg}$ but a dose of 0.75 $\mathrm{mg} / \mathrm{kg}$ results in increased dysphoria and ataxia with no increase in sedation. ${ }^{89}$

Ketamine has been used extensively and safely perhaps most commonly via the intramuscular (im) route. ${ }^{10}{ }^{11}$ Oral ketamine at a dose of $10 \mathrm{mg} / \mathrm{kg}$ has been shown more effective than placebo in the ED. ${ }^{12}$ It has the advantage of potent analgesia but there are concerns about vomiting and emergence phenomena. ${ }^{13}$

These two agents have been compared in the ED using a randomised controlled trial. McGlone ${ }^{14}$ demonstrated that im ketamine provided better tolerance to painful procedures and better parental acceptance than intranasal midazolam.

Using the oral route allowed us to conduct a double blinded trial. We chose to compare our normal dose of $0.7 \mathrm{mg} / \mathrm{kg}$ midazolam with ketamine $10 \mathrm{mg} / \mathrm{kg}$ as used by Qureshi. ${ }^{12}$

\section{Methods}

This trial was approved by our local hospital ethics committee.

Children aged 1 to 7 with lacerations requiring local anaesthetic (LA) injection before suturing were considered for the trial. If topical LA was to be used they were considered if their anxiety score was greater than one (see table 1). We excluded children requiring admission or opioid analgesia, those with known allergy, active respiratory disease, recent head injury, those taking erythromycin and refusal of consent. Recruitment was consecutive. Children were entered into the trial following parental consent then received the next trial drug solution, either midazolam $0.7 \mathrm{mg} / \mathrm{kg}$ or ketamine $10 \mathrm{mg} / \mathrm{kg}$.

Drug solutions were preprepared by the hospital pharmacy and numbered sequentially, 
Table 1 Anxiety/tolerance scores

\begin{tabular}{|c|c|c|}
\hline Score & Anxiety before & Tolerance during \\
\hline 1 & cooperative & cooperative/sleeping \\
\hline 2 & crying only when wound is touched & intermittent crying \\
\hline 3 & crying during general examination & continuous crying and/or additional restraint \\
\hline 4 & uncontrolled crying & uncontrolled crying and additional restraint \\
\hline
\end{tabular}

Taken from Preanesthetic medication in children. Anesthesiology 1992;76:28-33.

\begin{tabular}{ll} 
Table 2 & Sedation score \\
\hline Score & Level of sedation \\
\hline 1 & $\begin{array}{l}\text { barely rousable, asleep, needs shaking/shouting to } \\
\text { arouse } \\
\text { asleep, eyes closed, arouses with soft voice or light } \\
\text { touch } \\
\text { sleepy, eyes open but less active or responsive } \\
\text { awake } \\
4\end{array}$ \\
\hline
\end{tabular}

randomly containing one or other drug in concentrations such that $0.2 \mathrm{ml} / \mathrm{kg}$ of the solution delivered the correct dose. These were made up to $20 \mathrm{ml}$ with blackcurrent flavoured cordial. Ease of administration was judged by the parents as: no problem, some persuasion needed, or difficult. LA was administered when the sedation score, recorded every five minutes, was less than 4 (table 2) or at latest by 45 minutes after dosing.

Parents were used as observers for anxiety and tolerance and nurses and registrars for side effects. The primary outcome measures were:

1 Anxiety and tolerance scores (see table 1) before the procedure, during LA infiltration or application and during the procedure.

2 New behavioural disturbance (see table 2) within the first two weeks recorded by the parents and returned by postal questionnaire, chased up by telephone if required.

Secondary outcome measures were:

1 The time to reach a sedation score of less than 4 and time to recovery.

2 The occurrence of unwanted side effects during the ED stay and recorded by the parents during the first 24 hours. Observers were given a list of specific known side effects. Parents and children were followed up at five days.

3 Parental acceptance assessed qualitatively at five day follow up.

Procedures were performed by registrars in the resuscitation area using pulse oximetry monitoring with a nurse trained in paediatric life support present at all times. LA application and suture materials were standardised. We used $1 \%$ lignocaine (lidocaine) for injection and a gel containing adrenaline (epinephrine) $(1: 2000)$ and cocaine $(4.7 \%)$ was used for topical anaesthesia. Topical LA was not used for extremity wounds, those involving mucous membranes or lacerations longer than four centimetres.

Criteria for discharge were a return to base line verbal and motor skills. Parents were given a set of discharge instructions.

Results were analysed by our Research Support and Development Unit. Tolerance, anxiety and sedation scores and timings were compared using the Mann-Whitney $U$ test and side effect profiles using Fisher's exact test unless otherwise stated.
Table 3 Patient demographics

\begin{tabular}{lll}
\hline & Midazolam & Ketamine \\
\hline Number & 29 & 30 \\
Mean age & 4.1 & 4.1 \\
\% Male & 54 & 52 \\
\% LA injection & 46.4 & 51.7 \\
Median presenting anxiety score & 2 & 2 \\
Laceration & & \\
Mean length (cm) & 2.0 & 1.9 \\
Suturing time (median, min) & 10 & 10 \\
Location: \% head/face & 68 & 72 \\
\hline
\end{tabular}

\section{Results}

Fifty nine children were recruited. One patient in the midazolam refused the medication, going on to have wound repair using topical LA only. One patient in the ketamine group received the wrong dose. Table 3 shows the groups were not statistically different at presentation, demonstrating successful randomisation.

There was no difference in the ease of administration of either sedative using an intention to treat analysis with all 59 subjects.

An efficacy analysis excluding the two above patients showed better tolerance to LA application with ketamine than midazolam $(p=0.036)$. A sub-analysis showed that LA injection was better tolerated with ketamine (median tolerance scores 2 versus $3, p=0.029$, see figure 1 ) but no different when topical LA was used (median tolerance scores 2 and 2, $\mathrm{p}=0.10$ ). There was a trend for the ketamine group to have a lower tolerance score during suturing not reaching the $\mathrm{p}=0.05$ significance level (median tolerance scores 2 versus $1, \mathrm{p}=0.098$ ). When the injected LA and topical LA groups were analysed separately, this trend became stronger but did not achieve the $p=0.05$ level $(\mathrm{p}=0.069)$. An intention to treat analysis including all patients made no difference to the results.

Sedation scores were lower with ketamine (medians 2 versus 3, p=0.039) and time to a score of less than 4 and thus the dosing to LA injection was faster (medians, ketamine 20 minutes, range $14-55$, midazolam 43 minutes, range $5-50, p=0.001)$. Times from sedative administration to discharge were similar (105 and 110 minutes, range 60-265).

Dysphoric reactions were not reported with ketamine, but six reports of inconsolable agitation occurred in the midazolam group, two during the procedure, two in the recovery phase and two several hours later at home $(p=0.01)$. Vomiting occurred in six cases in the ketamine group (three in the ED, three at home) and two in the midazolam group in the ED, this difference was not significant $(p=0.14)$. There was significantly more nystagmus noted in the ketamine group but no reports of hypersalivation or laryngospasm. Despite strict discharge criteria, parents reported two cases in each group with ataxia, and drowsiness was more commonly reported with ketamine (4 versus $0, p=0.025$ ). Disturbed sleep on the first night occurred in three in the ketamine group and two in the midazolam group $(\mathrm{p}=0.36)$.

Oxygen saturations below $94 \%$ occurred in two patients in the midazolam group (90 and 


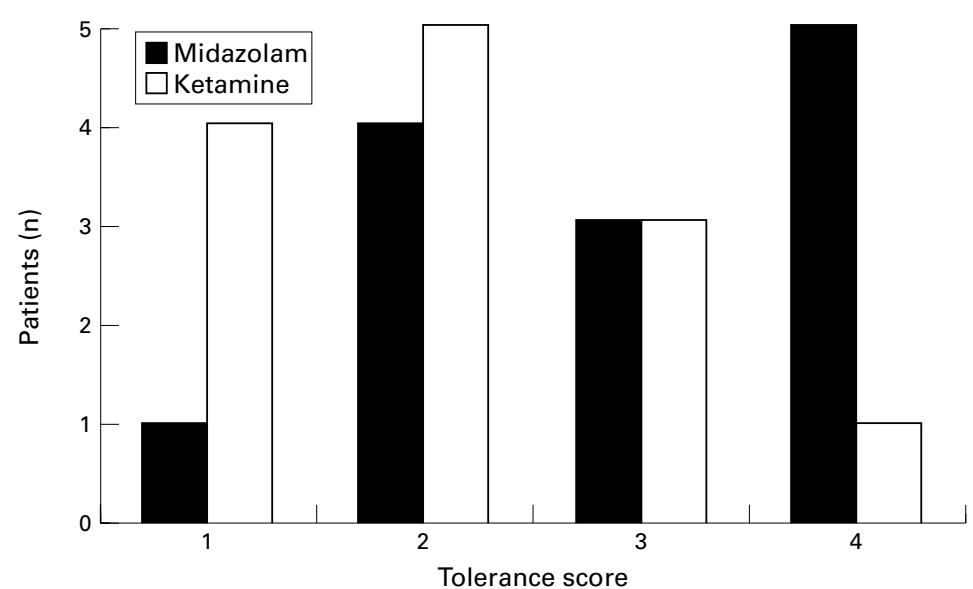

Figure 1 Tolerance to LA injection; better in the ketamine group. A higher tolerance score indicates a lower tolerance to injection.

Table 4 Behavioural changes within the first two weeks; more with midazolam. Parents asked about agitation, sleep disturbance, nightmares, bed wetting, temper tantrums, poor appetite, disobedience, separation anxiety

\begin{tabular}{lll}
\hline & \multicolumn{2}{l}{ Number of children } \\
\cline { 2 - 3 } Number of behavioural changes reported & midazolam & ketamine \\
\hline none & 13 & 21 \\
1 & 5 & 2 \\
2 & 2 & 3 \\
3 & 3 & 1 \\
4 & 2 & 0 \\
Total children reporting behavioural & 12 & 6 \\
\hline
\end{tabular}

$91 \%$ ) and in one ketamine patient (93\%). Overall lowest recorded oxygen saturations showed a trend to be lower with midazolam $(\mathrm{p}=0.058)$. There were no differences in respiratory or pulse rates.

Ninety one per cent of patients and parents returned to five day follow up. Of these 23 sets of parents found the procedure acceptable and not stressful, 22 acceptable and slightly stressful, seven just acceptable and very stressful and two (one in each group) not acceptable. There were no significant differences between groups $(\mathrm{p}=0.146)$.

Follow up for behavioural changes from 24 hours to two weeks was achieved in $92 \%$ of cases. Three parents in the midazolam and one in the ketamine group reported that their child remembered the procedure $(p=0.14)$. Thirty six per cent of parents reported one or more new behavioural changes. These were more commonly reported in the midazolam group $(48 \%$ versus $25 \%$, Mann-Whitney U $\mathrm{p}=0.0487, \chi^{2}$ test $\mathrm{p}=0.097$, see table 4 ).

\section{Discussion}

Our study demonstrated better tolerance to LA injection with ketamine measured by a commonly used four point behavioural score. ${ }^{6}$ We found no difference in tolerance when topical LA was used suggesting ketamine is only useful when LA injection is used. An efficacy analysis was thought more appropriate in this study. When an intention to treat analysis was performed with two further patients no difference in the results was found. A control arm with no sedation has been suggested for this study. We thought that this might be ethically difficult and varied significantly from our current practice such that it would not be acceptable to staff.

In this pilot study we did not perform a power calculation. This calculation cannot be performed when judging differences between treatments using a non-continuous ordinal scale. It is possible that a type II error occurred in the tolerance to procedure result. We could have measured anxiety and tolerance using a continuous visual analogue scale, which would permit a power calculation, avoid the possibility of a type II error and possibly increase the validity of a result. To detect a $20 \%$ difference with $90 \%$ power at the 0.05 significance level would require 105 patients in each group. We acknowledge that a larger study would more adequately answer this question.

Ketamine produces a unique dissociated state making assessment difficult. By recruiting the parents as observers we hoped to achieve a truly blinded result. Ideally we would have liked the luxury of an additional blinded observer.

Children may only communicate their distress after a traumatic experience by exhibiting behavioural changes. We asked about different aspects of behaviour and found a difference in the number of children exhibiting altered behaviour in favour of ketamine. When analysed by number of occurrences per child, thus taking severity of reaction into account, this was significant (Mann-Whitney $U$ test, $\mathrm{p}=0.048$ ) and a trend was found by overall $\chi^{2}$ analysis $(p=0.097)$. Although not concrete evidence, this suggests that those receiving midazolam were more likely to have a distressing experience. $^{2}$ McGlone $^{14}$ found im ketamine gave better tolerance to injection and procedure than intranasal midazolam. Follow up was to 72 hours and although no child was reported to remember the procedure behavioural change was not assessed. This area is not reported by Qureshi. ${ }^{12}$

The median time to adequate sedation with midazolam of 43 minutes was longer than other studies, ${ }^{8915} 16$ although these studies did not use a 5 point measurement scale. Time of onset with ketamine agreed with Gutstein ${ }^{17}$ and was shorter than the median 35 minutes reported by Qureshi ${ }^{12}$ using the sedation score. We noted wide ranges in time of onset of sedation, for example between 5 and 50 minutes for midazolam. Times to discharge were similar suggesting a longer recovery with ketamine and agreed with other reports.

The occurrence of emergence delirium is thought to be less common in children but its true incidence is not known. ${ }^{13}$ Along with Qureshi $^{12}$ we found no evidence of this phenomenon during the recovery period. In the midazolam group there were six cases of inconsolable agitation occurring in the first 24 hours. This reaction is described in most midazolam trials. ${ }^{6-11} 1415$ Its incidence is dose related and has been shown to be more common at an oral dose of $0.75 \mathrm{mg} / \mathrm{kg}$ compared with $0.5 \mathrm{mg} / \mathrm{kg} .{ }^{9}$ In retrospect we are concerned that using a dose of $0.7 \mathrm{mg} / \mathrm{kg}$ may 
have increased the incidence of this reaction thus affecting our results. Although these reactions occurred after LA injection we cannot exclude this possibility. We also feel that our reporting system for recovery behaviour was weak and that a descriptive ordinal scale or visual analogue scale could have been used.

Vomiting occurred in $20 \%$ of the ketamine group and appeared more frequent than in other reports. ${ }^{11}{ }^{12} 14$ The reported ataxia after dicharge emphasises the need for strict dicharge criteria and written and verbal advice for parents.

Only one other trial has reported a worrying desaturation $^{8}$ with midazolam. We found two cases with oxygen saturations of $90 \%$ and $91 \%$ recorded with pulse oximetry, recovering with stimulation and oxygen. There are reports of significant desaturations with im ketamine ${ }^{11}$ but the incidence with oral use is unknown. There were no reports of desaturations in the study by Qureshi ${ }^{12}$ In our study one patient's saturation decreased to $93 \%$, which responded to stimulation and oxygen. We note that continuous nurse and pulse oximetry monitoring is not universally recommended for oral midazolam in the $\mathrm{UK}^{18}$ but we strongly suggest it in the first hour when using either drug.

\section{Conclusions}

When LA injection is required in children aged 1 to 7 before wound repair, oral ketamine (10 $\mathrm{mg} / \mathrm{kg}$ ) provides better sedation than oral midazolam $(0.7 \mathrm{mg} / \mathrm{kg})$. This finding is strengthened by evidence of less behavioural change after ketamine within the first two weeks. Oral ketamine at this dose has a faster onset of sedation but times to discharge are similar. We can confirm other reports of paradoxical agitation with midazolam use. By using a dose over $0.5 \mathrm{mg} / \mathrm{kg}$ these events may have been more frequent and affected our results. Both drugs may cause oxygen desaturation and we recommend nurse and pulse oximetry monitoring for one hour after sedative administration. Further studies are needed to define the optimum doses of both agents and more accurately compare their effect on tolerance to procedures.
Contributors

Paul Younge and Jason Kendall conceived the idea for the study hypothesis and the details of the study design. The day to day running of this study and data collection was organised and supervised by Paul Younge. The hospital pharmacy at Frenchay Hospital prepared the study materials and all data were analysed by the Research Support Development Unit at Frenchay Hospital. The paper manuscript was prepared by Paul Younge and edited by Jason Kendall.

Funding: none.

Conflicts of interest: none.

1 Kendall JM, Charters A, Mccabe SE. Topical anaesthesia for children's lacerations : an acceptable approach. F Accid Emerg Med 1996;13:119-22.

2 Vernon DTA, Schulman JL, Foley JM. Changes in children's behaviour after hospitalisation. Am $\mathcal{F}$ Dis Child children's behaviour

3 Eckenhoff JE. Relationship of anaesthesia to post operative personality changes in children. Am $\mathcal{F}$ Dis Child 1953;86: 587-91.

4 McClusky A, Meakin GH. Oral administration of midazolam as a premedicant for paediatric day-case anaesthesia. Anaesthesia 1994;49:782-5.

5 The Pediatric Committee of the American College of Emergency Physicians. Paediatric Analgesia and Sedation. Ann Emerg Med 1994;23:237-50.

6 Hennes HM, Wagner V, Bonadio W, et al. The effect of oral midazolam on anxiety of preschool children during laceramidazolam on anxiety of preschool children

7 Taiwo B, Flowers M, Zoltie N. Reducing children's' fear when undergoing painful procedures. Arch Emerg Med 1992;9:306-9.

8 Davies FC, Waters $M$. Oral midazolam for conscious sedation of children during minor procedures. 7 Accid Emerg Med 1998;15:244-8.

9 McMillian CO, Sparth-Schopfer IA, et al. Premedication of children with oral midazolam. Can f Anaesth 1992;39:54550.

10 White PF, Way WL, Trevor AJ. Ketamine-its pharmacology and therapeutic uses. Anesthesiology 1982;56:119-36.

11 Green SM, Rothrock SG, Lynch EL, et al. Intramuscular ketamine for pediatric sedation in the emergency department: safety profile in 1,022 cases. Ann Emerg Med 1998;31:688-97.

12 Qureshi FA, Mellis PT, McFadden MA. Efficacy of oral ketamine for providing sedation and analgesia to children requiring laceration repair. Pediatr Emerg Care 1995;11:937.

13 Sussman DR. A comparative evaluation of ketamine anesthesia in children and adults. Anaethesiology 1974;40: 459-64.

14 McGlone RG, Ranasinghe S, Durham S. An alternative to "brutacaine": a comparison of low dose intramuscular ketamine with intranasal midazolam before suturing. 7 Accid Emerg Med 1998;15:231-6.

15 Weldon CB, Watcha MF, White PF. Oral midazolam in children: effect of time and adjunctive therapy. Anesth Analg 1992;75:51-5

6 Malinovsky JM, Populaire C, Cozian A, et al. Premedication with midazoalm in children. Effect of intranansal, rectal and oral routes on plasma midazolam concentrations. Anaesthesia 1995;50:351-4.

17 Gutstein HB, Johnson KL, Heard MB, et al. Oral ketamine preanesthetic medication in children. Anesthesiology 1992; 76:28-33.

18 Walker J. the use of oral midazolam in Accident and Emergency to reduce anxiety in children. Accid Emerg Nurse 1996;4:110-13. 